\title{
La Revista Latinoamericana de Estudios Educativos
}

\section{The Latin American Journal of Educational Studies}

Sylvia Schmelkes*

La historia de la Revista Latinoamericana de Estudios Educativos se encuentra íntimamente ligada a la del Centro de Estudios Educativos (CEE), fundado por Pablo Latapí Sarre en 1963, como una Asociación Civil, no lucrativa, dedicado a la investigación de la problemática educativa de México y de América Latina. Durante los primeros ocho años llevó el nombre de Revista del Centro de Estudios Educativos, aunque su vocación explícita fue ser una vía para la difusión de la investigación educativa realizada en América Latina en tiempos en los que ésta era escasa. El CEE fue pionero en la investigación educativa en el país y modelo para otros centros similares, como el Centro de Reflexión y Planificación Educativa (CERPE) de Venezuela y el Centro de Investigación y Desarrollo de la Educación (CIDE) de Chile, fundados poco después.

La Revista, con 48 años de historia, se encuentra también estrechamente vinculada a la historia de México a lo largo de esos años, y muy particularmente a la historia de su educación. Y porque así se lo propuso, su desarrollo da cuenta de la evolución de la investigación educativa en el país y en América Latina.

En este artículo nos proponemos hacer un breve recuento, a vuelo de pájaro, de la evaluación de la Revista y de la manera como se ha propuesto reflejar estos procesos.

El Centro de Estudios Educativos, en sus primeros años, tuvo tres ediciones periódicas: Cuadernos de Divulgación, que ponían en circulación artículos y documentos interesantes publicados, en su mayoría, en otros países; Boletín Estadístico, que presentaba la recopilación que por primera vez se hacía de la estadística funda- 
mental del Sistema Educativo Nacional; y Noticias y Comentarios, que reunía y comentaba críticamente los acontecimientos educativos fundamentalmente del país. En 1971 Pablo Latapí propone fusionar estas publicaciones y dar un importante paso a la publicación de la primera revista científica en materia de investigación educativa del país. En la editorial del primer número se presenta su propósito:

La Revista del Centro de Estudios Educativos intenta ser una modesta contribución al diálogo y la cooperación en materia educativa entre los países de América Latina, proporcionando un medio especializado de expresión a todos aquellos que estén interesados en esta problemática. La Revista no está cerrada a ningún tipo de ideas o ideologías: se abre a todo esfuerzo por plantear seriamente nuestros problemas reales.

No estaba lejos el movimiento estudiantil de 1968, que impactó fuertemente al CEE. Por eso quizá desde el primer número muestra su preocupación por la justicia educativa y su vocación latinoamericana. Adolfo Mir escribe un artículo sobre los determinantes económicos de las desigualdades estatales en los logros educativos en México. José Cen Zubieta, Pablo Latapí y Francisco Patiño presentan un modelo para la localización de escuelas y el diseño del transporte escolar. Carlos Muñoz Izquierdo, investigador principal del CEE desde sus inicios y hasta 1984, escribe sobre la productividad de la educación en México. La Revista se organiza en una sección de artículos, otra de informes y documentos, una sección estadística (que retoma el propósito del antiguo Boletín Estadistico), y una sección de reseñas de libros.

En el número 2 del primer año se incluye el Estudio de evaluación de las Escuelas Radiofónicas de la Tarahumara, respuesta del CEE a la petición de la misión jesuita en la Tarahumara, para conocer el impacto de escuelas radiofónicas creadas en 1954.

La Revista es rápidamente acogida por investigadores de la educación de México y de América Latina, así como por investigadores de otras regiones que investigan a América Latina. En el primer volumen ya aparecen, además de un artículo de Carlos Muñoz Izquierdo, notable investigador del CEE, escritos de reconocidos investigadores como Roland Paulston, Martin Carnoy, 
Ernesto Meneses, David Barkin, Ernesto Schiefelbein, Gabriel Cámara, entre otros. Ya en el número 3 de este primer volumen se inaugura en la Revista una sección de comunicaciones, pues Aldo Solari comenta el artículo de Paulston en el número anterior y comienza el diálogo académico.

En el editorial del número 3 de este primer volumen, el CEE adopta una postura teórica para entender la relación entre sociedad y educación. Tras una crítica al funcionalismo de Durkheim y Parsons, argumenta que la sociología del conflicto es la que permite entender el papel que juega la educación en una sociedad clasista dominada por el capitalismo como la de México: un papel reproductor de los privilegios de unos cuantos. Este marco teórico prevalecerá en sus investigaciones a lo largo de más de una década.

En el segundo volumen de la revista se pone de manifiesto su función de órgano de difusión de la investigación educativa en América Latina. En él hay trabajos sobre Argentina, Brasil, Chile, Colombia y Venezuela, además de México. Por la temática de los artículos, la Revista adquiere su personalidad como divulgadora de investigación de economía y sociología de la educación, y no pedagógica, al menos no en esta primera época. Ello, a su vez, es reflejo de la orientación del propio CEE, cuya preocupación fue, desde el inicio, el papel social y económico de la educación. En el editorial del primer número del segundo volumen se reitera la orientación teórica del conflicto, pero se añade la perspectiva valorativa, centrada en la justicia social. Ello también habrá de marcar la orientación de la Revista a lo largo de su historia.

En el número 3 del volumen II se anuncia el retiro de Pablo Latapí de la dirección del CEE, y con ello el fin de su primera etapa. Con ocasión de este cambio, el personal del CEE llevó a cabo un mes de autoestudio en el que revisó y evaluó once años de existencia. Reafirma su vocación de prestar un servicio a la sociedad mexicana y latinoamericana mediante la investigación científica de los problemas educativos. Y se inclina por un enfoque de cambio estructural para interpretar la realidad educativa y orientar su quehacer. Rechaza el desarrollismo y abraza la teoría de la dependencia. Y desde ahí, cuestiona "la educación para el desarrollo" y entiende el papel que se desea de la educación como 
uno que favorece el cambio social que se ve como necesario e inevitable. Dicho cambio, si bien supone superar la injusticia y la opresión, y por lo mismo el conflicto, debe orientarse a la reconciliación de las partes en conflicto, en aras de un humanismo profundo que persiga la paz y repruebe la violencia. La educación para el cambio debe capacitar a las clases dominadas para plantear las demandas y los conflictos para conquistar sus derechos, mediante el desarrollo de su capacidad crítica y de disentimiento. También, sin embargo, se veía necesario realizar un trabajo educativo con las clases dominantes para despertar su conciencia sobre los problemas sociales y convencerlos de la necesidad de transformar la sociedad hacia una mayor justicia.

La adopción de este marco de referencia no se oponía a la investigación científica. El compromiso del investigador es necesario; su objetividad lo define como científico. La institución lo procura, pero también, como se indica en la editorial del siguiente número (III-2), la obliga a plasmar en acciones concretas las 286 nuevas formas de educación.

El tercer volumen de la Revista es importante porque comienza a dibujarse el resultado de un gran esfuerzo de propuesta de Reforma Educativa que asumió el CEE en respuesta a la invitación del entonces presidente Luis Echeverría a la sociedad mexicana a hacer propuestas sobre la reforma de la educación. Este proyecto asume el marco de referencia esbozado desde 1972, enriquecido en las editoriales de este volumen, en el que además se asume la necesidad de plasmar en acciones concretas nuevas formas de educación orientadas a la transformación social.

El primer número de este volumen cuenta con un artículo de Robert Myers sobre la estructura ocupacional; con otro de Fernando Mockemberg que informa del estudio longitudinal de niños desnutridos en México. Pablo Latapí comenta el Informe Faure y José Teódulo Guzmán describe la educación indígena en Chiapas, ahora con referencia a la misión de los jesuitas en Bachajón. En el segundo aparece un informe de Olac Fuentes, y el CEE expone su juicio crítico sobre la obra educativa de Luis Echeverría.

Es en el número tres en el que se da cuenta del macroproyecto de Reforma Educativa del CEE, que se convierte en un trabajo 
multicitado tanto en México como a nivel internacional. Este proyecto revisa las relaciones entre la educación y el sistema económico, entre la educación y el sistema político, entre la educación y el sistema de estratificación social; analiza los valores en los que forma y debiera formar e incursiona en la educación alternativa por la vía de la educación popular para poblaciones marginalizadas. En él escriben los miembros del equipo del CEE en ese momento: Carlos Muñoz Izquierdo, Fernando Estrada Sámano, Rodrigo Medellín, Jorge Muñoz, José Teódulo Guzmán y Sylvia Schmelkes.

El volumen cierra con un número en el que se da cuenta de los centros de investigación educativa en América Latina, muchos de ellos creados a imagen y semejanza del CEE, como resumen de un seminario convocado por el propio Centro, que cristaliza la red incipiente de centros de investigación educativa latinoamericanos favorecida parcialmente por la Revista, y fortalece su accionar futuro.

Termina por entonces el tiempo de las políticas proteccionistas y el desarrollo equilibrado y comienza a vislumbrarse una tibia globalización en los países latinoamericanos. América Latina, y sobre todo el Cono sur, entra en una época oscura de cancelación de la democracia y de dictaduras militares represivas. México, en cambio, administra su abundancia petrolera, a pesar de lo cual la educación no promueve la equidad y ni la justicia social, y esta preocupación está presente en los investigadores que se expresan en la Revista. La época de abundancia se refleja en el CEE, que crece de 35 personas a 130 .

Durante los ańos siguientes sigue una fuerte presencia latinoamericana en las secciones de artículos y de informes de la Revista. Destacan Marcela Gajardo, Germán Rama, Víctor Urquidi, Miguel Escotet, Claudio de Moura y Castro. Es también espacio aprovechado por investigadores, sobre todo norteamericanos, que estudian la educación en América Latina y que quieren que los latinoamericanos conozcan los resultados de sus investigaciones, como Thomas La Belle, Martin Carnoy y Noel McGinn. Carlos Lenkersdorf publica lo que quizás es su primer trabajo sobre los tojolabales en 1975. Aunque la Revista no es temática, el número 2 de ese año se dedica casi exclusivamente a la educa- 
ción radiofónica, pues recoge los trabajos de un seminario sobre el tema también convocado por el CEE.

En el editorial del primer número del volumen VI, el CEE hace un análisis de las tendencias reproductoras de la educación en México y del papel de los grupos independientes en la educación, lo que fundamenta las prioridades del CEE a futuro:

Del análisis anterior, el CEE ha deducido, para el desarrollo futuro de sus investigaciones, las siguientes áreas prioritarias:

1. Investigación de las relaciones entre educación y sociedad ...

2. Evaluación y diseńo de innovaciones educativas... no formales...y formales.

Además se propone realizar seminarios de reflexión sobre las experiencias de los educadores independientes y aumentar la difusión de las investigaciones y de los seminarios de reflexión.

Desde este volumen, cada tercer número incluye los comentarios sobre el apartado educativo de los informes presidenciales de México -material riquísimo para el análisis histórico de la política educativa-; cada número IV incluye un informe de actividades del CEE, además de, intermitentemente, un índice del volumen de gran utilidad para pronta referencia.

Dadas las prioridades enunciadas, la Revista comienza a ser espacio de escritos y estudios sobre educación rural y no formal, aunque también sigue reflejando los resultados de la investigación latinoamericana sobre educación superior. El editorial del primer número del volumen VII se dedica a la educación no formal y el quehacer científico.

Conforme se van incorporando nuevos miembros al CEE -siempre fue un semillero de investigadores-, van apareciendo como autores de artículos, ensayos o informes, además del equipo inicial, ya mencionado, Mario Pacheco, Jorge Martínez Sánchez, Bertha Salinas, Pedro Gerardo Rodríguez, Enrique Luengo, Cristina Casanueva. Esta tendencia continúa a lo largo de la historia de la revista. Se mantiene la presencia de investigadores latinoamericanos, y sigue siendo espacio para estudiosos mexicanos como Víctor Urquidi y José Ángel Pescador. 
En 1978 cobra importancia mundial la discusión sobre la importancia del contexto y de la escuela en los resultados y las desigualdades educativas. Esta discusión se refleja en el número 2 del volumen VIII, en la que se plantean tres teorías: las que enfatizan los factores del ámbito escolar, las que atribuyen las desigualdades a factores culturales, y las que consideran que dichos resultados y desigualdades se deben principalmente a los condicionamientos estructurales externos a la escuela y al sistema escolar. El CEE se pronuncia por darle mayor credibilidad a los factores culturales y estructurales.

El editorial del primer número del vol. IX se dedica a celebrar los 15 primeros años del CEE. Reitera el marco axiológico que ha inspirado sus actividades: la lucha por la justicia social, que a su vez exige una transformación estructural de manera que puedan desarrollarse y compartirse los valores inherentes a la persona humana. Este volumen también celebra los primeros ocho años de la Revista, que a esa fecha había publicado 101 artículos científicos sobre temas económicos, sociales, culturales y políticos relacionados con la educación, además de innumerables informes, ensayos, documentos, comunicaciones y estadísticas sobre el estado actual de la educación en México y en América Latina. Este año cambia su nombre a Revista Latinoamericana de Estudios Educativos, por considerar que en sus ocho años de existencia había logrado "el diálogo y la confrontación abierta entre investigadores de la educación latinoamericana para proponer planteamientos propios y metodologías congruentes con la problemática de nuestros países" (Editorial, 1979, p. VIII).

El segundo número de este volumen incluye un artículo de Pablo Latapí sobre las prioridades de la investigación educativa en México, que presenta por vez primera un pronunciamiento del aparato de apoyo al quehacer científico del país (Conacyt) sobre lo que debía ser investigado en torno a la educación del país.

Este volumen incluye artículos de investigadores de renombre como Aldo Solari, Daniel Levy, Jacques Velloso, Abraham Magendzo, Roland Paulston, y se siguen incorporando investigadores recién llegados al CEE, como Guillermo González Rivera, Andrés Peraza y Andrés Sotelo. Este volumen también es testigo de las primeras discusiones sobre derechos humanos y educa- 
ción en una reseña sobre un seminario interamericano en torno al tema. El cuarto número de este volumen incluye un artículo seminal de Carlos Muñoz Izquierdo, titulado "El síndrome del atraso escolar y el abandono del Sistema Educativo". Su importancia radica en que abre el espectro de la investigación que realiza el CEE a los factores escolares que influyen en los resultados educativos. Con ello comienza, creo, la orientación sociopedagógica de una parte de la investigación del CEE, que influye junto con otros factores, en la investigación que se realizará en adelante en América Latina.

El volumen X, en 1980, contiene artículos de intelectuales de diversas nacionalidades como Noel McGinn, Carlos Alberto Torres, Carlos Payán, Bernardo Toro, Marco Navarro, Donald Snodgrass. Eugenio Maurer, investigador del CEE, escribe sobre la política educativa indigenista y Sylvia Schmelkes sobre la educación rural. Se da cuenta del surgimiento de otras revistas de investigación educativa en América Latina: Educación, de Cuba y Educación Hoy, de Colombia. El editorial del primer número presenta algunos apuntes teóricos para evaluar el impacto de diferentes tipos de investigación educativa, con lo que inicia la preocupación por la relación entre investigación, política pública y cambio educativo.

En 1981 se cumplen diez años de la Revista. El editorial de su primer número reflexiona sobre lo publicado hasta la fecha, $\mathrm{y}$ considera que si bien los estudios son de diversa índole y con variada orientación, en todos ellos puede observarse un énfasis en "la necesidad de clarificar el papel de la educación en la conservación de estructuras sociales injustas, o en las transformación social, política y económica que permita ir avanzando hacia sociedades más justas y equitativas" (Editorial, 1981, p. 5). Clasifica las publicaciones de la Revista en los siguientes rubros: 1) estudios con base empírica directa sobre la relación entre el ámbito educativo y el social, político y económico; 2) 829 estudios sobre los determinantes valorales y actitudinales de la educación y los efectos de ésta sobre en la transformación de actitudes y valores (ámbito psicosocial); 3) estudios sobre el funcionamiento interno de los sistemas educativos formales y no formales; 4) artículos o ensayos sobre el papel de la educación y la investigación en el proceso de 
desarrollo o cambio social, y 5) presentaciones analíticas y propositivas sobre metodologías y técnicas de investigación. Concluye que "la comunidad de investigadores sobre América Latina ... no considera el fenómeno educativo como algo que deba estudiarse aislada y académicamente. Lo fundamental es su interacción con fenómenos sociales más amplios" (Editorial, 1981, p. 7).

La década de los ochenta es tiempo de crisis económica en toda la región -la década perdida-. El CEE no es la excepción. El personal se redujo de 130 a 60 personas, y luego nuevamente a 30. La investigación sufre la crisis y muchos centros de investigación social pierden los apoyos tradicionales que procedían de fundaciones y se ven en la necesidad de vender proyectos para sobrevivir. No obstante, durante esta época el CEE es consciente de la necesidad de ser selectivos en los proyectos que acepta, y de documentar científicamente para compartir los resultados de cada uno. Algunos de ellos se verán reflejados en la Revista a lo largo de la década.

El CEE comienza a llevar a cabo proyectos de intervención educativa de importante repercusión en la región. El Proyecto Nezahualpilli es uno de ellos, que lleva la educación preescolar con enfoque constructivista, a través de madres educadoras, a una zona urbana muy marginal -Ciudad Nezahualcóyotl-. Otro más es un modelo alternativo de educación secundaria para zonas rurales, que experimenta de manera pionera con proyectos que integran las diversas áreas disciplinares en "Unidades de Aprendizaje-Investigación-Acción”. De ambos da cuenta esta década. Pablo Latapí publica un artículo muy consultado sobre la alfabetización en seis países de América Latina. Esta década reporta contribuciones de Felipe Martínez Rizo, Norberto Fernández Lamarra, Luis Porter, George Psacharopolus, José Joaquín Brunner, María de Ibarrola, Etelvina Sandoval, Henry T. Trueba, entre muchos otros. Entre los colegas investigadores del propio CEE destacan Lautaro Prado, Luis Narro, Sonia Lavín, Cecilia Fierro, Rolando Maggi, Lesvia Rosas y Flavio Rojo.

En 1986 la Revista cumple 15 ańos, y el editorial se refiere a ella como un órgano que ha podido dar a conocer investigaciones importantes realizadas en la región y servir como espacio para difundir pensamiento crítico sobre la teoría y la práctica educa- 
tiva a nivel latinoamericano. La intención ha sido, dice, ofrecer al público informado en América Latina un producto periódico de alta calidad con contenidos con valor científico. Considera que ha venido creciendo el rigor científico de la investigación realizada en América Latina. Los centros de formación de investigadores de alto nivel en ciencias de la educación incluyen la Revista en sus acervos bibliográficos, y sus piezas se encuentran en las bibliografías de sus cursos. Es difícil encontrar una investigación de envergadura en América Latina que no la refiera. Se señala que ya las contribuciones hacen la masa crítica suficiente como para elaborar estados del arte sobre los temas que se han presentado. Hace ver que alrededor de una tercera parte de las contribuciones de la Revista son de trabajos realizados en México. Los escritos sobre América Latina tuvieron un auge entre 1976 y 1980 . Colombia y Chile son los que han contribuido con mayor número de escritos, pero también destaca la presencia de Brasil, Argentina, Perú y Venezuela. El tema más frecuente es la educación superior, seguido muy de cerca por el de economía de la educación. Pero también destacan las temáticas de sociología de la educación, educación y política, educación básica y sistemas educativos. Por el contrario, los que han recibido poca importancia es la educación preescolar, la educación técnica y la capacitación, el magisterio, el currículum, la psicología educativa, la educación indígena y la historia de la educación.

En 1988, el CEE cumple 25 años. De la reseña que hace el editorial de la Revista destaco lo siguiente: si bien en los primeros años los destinatarios de su quehacer eran fundamentalmente los investigadores y los tomadores de decisiones, algunos ańos más tarde se añaden los grupos marginalizados y se comenzó a privilegiar la educación no formal de adultos al lado de la formal. Se señala este como el cambio más profundo experimentado por el CEE en estos primeros 25 ańos. Ello no se hizo a costa del rigor académico, que desde un principio prestigió su trabajo, ni al énfasis interdisciplinario que se señaló en sus orígenes como una ruta para la investigación educativa en el país. Se siguió vinculando la investigación teórica con la aplicada, y se procuró en todo momento responder oportunamente a los retos de un entorno cambiante. El CEE ha contribuido a fortalecer la sociedad 
civil con su aportación desde la investigación, como actor y como efecto. Reitera su opción por la justicia y por las clases oprimidas.

Con motivo de los 25 años del CEE, este número de la Revista recoge "estados del arte" sobre lo que el CEE ha producido a lo largo de su historia en torno a planeación educativa; educación, producción y empleo; educación en el medio rural; investigaciónacción y formación de docentes en ejercicio.

Durante el siguiente lustro la Revista continúa con su carisma y su misión. La presencia latinoamericana disminuye, pues surgen revistas especializadas en varios de estos países que recogen su producción académica sobre educación. No obstante, publican en estos años Jorge Padua, Susan Street, María Antonia Gallart, Ángel Díaz Barriga, Justa Ezpeleta, José Rivero, Teresa Bracho, Fernando Reimers, Norbert Francis, Enrique Pieck, Guadalupe Díaz Tepepa. Bertha Salinas escribe sobre Educación Popular. Con motivo de los 500 ańos del encuentro entre dos mundos, la Revista se centra en la educación indígena.

En 1990 asume la dirección del Centro de Estudios Educativos Luis Morfín López.

El editorial del número 4 de 1992 (volumen XXII) se refiere al reconocimiento por parte de nuestras sociedades políticas como países pluriétnicos y multiculturales "con la proyección, al hacerlo, de una imagen de futuro de un país unificado a partir de la interacción enriquecedora entre grupos culturalmente diferentes" (Editorial, 1992, p. 6). Ubica la actividad educativa con indígenas en el centro de este cambio de concepción. Refiere al respecto las posiciones extremas: los asimilacionistas y los aislacionistas. Sostiene, sin embargo, que asumirnos como países pluriétnicos y multiculturales supone

asumir intencionalmente la necesidad de conocer, comprender y letrar las culturas indígenas en su propia lengua y la adquisición de una identidad cultural a partir de la cual se pueda, con fuerza, interactuar con otras, incluyendo la dominante... y participar activamente en la vida económica, social, política y cultural nacional (Editorial, 1992, p. 7).

Este año también ocurre una reforma educativa importante en México, con el Acuerdo Nacional para la Modernización de la 
Educación Básica y Normal, que además de conducir a una reforma curricular importante, descentraliza estos niveles educativos hacia las entidades federativas. Aunque no de forma inmediata, ello repercutirá en las investigaciones que realiza el CEE y otros investigadores nacionales y que se difunden a través de la Revista.

En 1993 la Revista publica el clásico artículo de Pablo Latapí sobre la Justicia en Educación. En ese mismo año, el CEE cumple 30 años, poco después de lo cual, en 1994, sobreviene una crisis en la institución. Los investigadores fundadores del CEE -Pablo Latapí, Carlos Muñoz Izquierdo, Manuel Ulloa y Sylvia Schmelkes-, se retiran como consecuencia de la misma. Comienza el CEE una nueva etapa.

Las editoriales de la Revista comienzan a ser piezas firmadas por el Director General. Sigue la tendencia de disminución de la presencia latinoamericana. Ya en el volumen XXVIII, todos los autores son mexicanos, y en el XXX todos los escritos son firmados por investigadores del CEE. La Revista, que había logrado su sitio en el padrón de revistas de excelencia en Conacyt, pierde esta distinción. Comienza a dejar de haber editoriales en algunos números, y en su lugar hay presentaciones del contenido. Se juntan las secciones de artículos y ensayos por ausencia de investigaciones empíricas. Comienzan a salir números dobles, en ocasiones dos veces al año, de manera que la periodicidad se vuelve bianual en lugar de trimestral.

El volumen XXXV celebra los 35 años de la revista en un número doble que recoge los artículos considerados "clásicos" de su pasado en una antología que contiene artículos de Schmelkes ("La Desigualdad en la Calidad de la Educación Primaria"); Lavín ("Centros de Educación Básica Intensiva: Una Alternativa al Rezago Escolar"); McGinn y Porter ("El Supuesto Fracaso de la Planificación Educativa en América Latina”); Pérez Alarcón, Abiega, Pamplona y Zarco ("Alternativas de Educación Preescolar para Zonas Marginalizadas Urbanas: El Proyecto Nezahualpilli"); Ávila ("El Saber Matemático de los Analfabetos: Origen y Desarrollo de sus Estrategias de Cálculo"), Muñoz Izquierdo, Rodríguez, Restrepo y Borrani ("El Síndrome del Atraso Escolar y el Abandono del Sistema Educativo"). 
Este número sí inicia con una editorial, firmada por Félix Francisco Martínez Rodríguez, que anuncia el inicio de una nueva época de la Revista, que busca continuar el diálogo como herramienta de construcción de la política pública, aspira a "construir los espacios ciudadanos para apuntalar la acción educativa como acción del Estado". Se propone explícitamente flexibilizar los criterios editoriales para facilitar la publicación de material. Las nuevas secciones son las siguientes:

- Para la agenda educativa nacional, donde se expondrán temas que deben conformar la agenda nacional en política educativa.

- Artículos y ensayos.

- Para la construcción de saber, donde se dará cabida a los trabajos cuya metodología sea ilustrativa de cómo abordar las cuestiones educativas hoy en día.

- Diálogo informado, espacio sobre la situación del sistema educativo

- Construcción ciudadana de lo público, donde se publicarán sugerencias ciudadanas de políticas y acciones a realizarse en materia educativa.

- De la historia para el presente, donde se incluirán artículos ya publicados de temas aún vigentes.

- Que leer. Reseñas de libros.

Esta nueva época le da a la Revista nuevos bríos. Los números están mucho más nutridos, aunque siguen siendo números dobles. En algunos números no aparece una sección de artículos y ensayos.

Y de esta manera se arriba a los 50 años del CEE, en el año 2008. La editorial formula las etapas por las que ha pasado la institución a lo largo de su historia:

- La primera, de 1963 a 1974, se guía por los supuestos siguientes: la educación es una variable importante en el desarrollo nacional; la información objetiva sobre el funcionamiento del Sistema Educativo genera una opinión pública madura; la comunidad académica y la opinión pública bien informada son los principales interlocutores de la producción del CEE. Por eso comienza su actividad con un diagnóstico de la educación en México.

- La segunda etapa, según este análisis, dura sólo dos años: 
1974-1976, se orienta por la convicción de que la relación entre educación y sociedad no es armónica y de que la educación no produce el cambio social por sí sola, sino que hay que incorporar otras variables económicas, políticas y sociales. Se valoran esfuerzos innovadores de la educación no formal.

- En la tercera etapa (1976-1983) el CEE introduce el "saber hacer", además del "saber", mediante proyectos de investigación-acción y considera que por medio de la acumulación incremental de cambios en grupos populares puede producirse un cambio cualitativo en la sociedad. Los sectores populares se definen también como interlocutores del quehacer del CEE.

- La cuarta etapa (1984-1990) asume los supuestos de que, si bien la educación es una variable "condicionada" por factores sociales, políticos y económicos, también puede ser una variable "condicionante" de los procesos de transformación social. Se revalora el papel de la educación.

- En la quinta etapa (1991-2013) se concibe la educación de calidad como un derecho de todo ser humano y como el medio para llegar a ser persona. Su campo prioritario de incidencia es la base cultural de la estructura social: la persona, la escuela, el alumno, el maestro, los padres de familia, y busca la emergencia de la ciudadanía. El campo privilegiado de la investigación del CEE se define como el tejido social y la organización desde la base.

Se reconoce que el CEE se ha mantenido como centro independiente a pesar de grandes crisis. Otros centros hermanos en América Latina, como el CERPE de Venezuela, o el CIDE de Chile, han sido absorbidos por universidades jesuitas en sus respectivos países.

Los últimos cinco años de vida del CEE se caracterizan por una nueva crisis, de naturaleza financiera nuevamente, que lo llevan a deshacerse de sus haberes, como la casa y la biblioteca, que en su momento fue la mejor biblioteca especializada en educación del país. También se desprende de la Revista. En el número doble 3 y 4 de 2017, su nueva directora, Annette Santos, firma una editorial en la que destaca el papel de la Revista de haberse puesto, a lo largo de 47 años, "al servicio de la construcción de una 
sociedad más justa e igualitaria, donde la calidad de la educación que reciben las personas no esté determinada por las condiciones de sus hogares ni del contexto en que viven" (Editorial, 2017, p. 6). Reconoce que la sociedad aún es injusta y desigual, y que la educación sigue jugando un papel reproductor de las desigualdades. No obstante, esto no desalienta. Por el contrario, "nos invita a mantener la tenacidad y la constancia, a la vez que allegarnos de mayores recursos e inteligencias para que tales esfuerzos logren impactos más fructíferos".

Anuncia en este escrito que este número doble es el último que corre a cargo de la institución que le dio origen. Explica que el CEE comienza un proceso de refundación para poder seguir siendo una institución académica independiente comprometida con la promoción de la justicia, la libertad y la democracia a través de la educación, en particular de aquella destinada a las poblaciones más desfavorecidas.

La Revista pasa ahora a ser responsabilidad del Campo Estratégico de Acción en Modelos y Políticas Educativas del Sistema Universitario Jesuita, con la Universidad Iberoamericana Ciudad de México a la cabeza. La idea es convertirla en un órgano de difusión de esta red que promueve la justicia social a través de la educación.

\section{REFLEXIÓN FINAL}

Hemos hecho un resumen breve y necesariamente parcial de un órgano de difusión de la investigación educativa que se caracteriza por tres rasgos: 1) una clara orientación científica; 2) una aproximación interdisciplinaria a la comprensión del fenómeno educativo, y 3) un definido compromiso hacia el fortalecimiento de la comprensión y del impacto de la educación en su contribución hacia una mayor justicia social, lo que la hace probablemente única en su género.

La Revista fue pionera en su género en la región latinoamericana, a la que sirvió de espacio de difusión y discusión del conocimiento generado sobre la educación en la región durante al menos dos décadas. Ha permanecido fiel a su impronta de origen 
gestada por su fundador, Pablo Latapí Sarre, a lo largo de 48 años ininterrumpidos.

Durante su larga historia, la Revista ha reflejado los cambios políticos y educativos de la región, y ha intentado comprender y dar respuesta a los nuevos retos educativos producto de las crisis económicas, de las dictaduras políticas, de los errados modelos de desarrollo de nuestros países. Ha dado seguimiento preciso a las políticas y reformas educativas de México y de la región latinoamericana, así como de la evolución de la manera como se cumple en nuestros países el derecho a la educación.

A través de sus editoriales, así como de muchos artículos, ensayos y notas diversas, la Revista ha sido una voz de reflexión profunda, de denuncia y exigencia de cambios educativos y sociales, pero también de importantes propuestas educativas probadas como exitosas, orientadas en su mayoría a la educación de los más necesitados.

Sería interesante un estudio de impacto del casi medio siglo 298 de su existencia sobre la política educativa en México y en la región. Sin duda lo ha tenido, aunque la evidencia al respecto es elusiva. De lo que no hay duda alguna es de su impacto sobre la investigación educativa -referencia obligada para las investigaciones importantes que se realizan sobre la región-, así como sobre la formación no solamente de investigadores educativos, sino de educadores y tomadores de decisiones en el campo. Probablemente también haya contribuido a aportar una orientación valoral, centrada en la educación para la justicia, a la investigación educativa que se realiza en nuestra región.

Los propósitos iniciales y permanentes de la revista coinciden con los del Sistema Universitario Jesuita, y especialmente con el Campo Estratégico de Acción en Modelos y Políticas Educativas, ahora liderado por la Universidad Iberoamericana Ciudad de México. Confiamos en que en estas manos seguirá persiguiendo la difusión del conocimiento generado con rigor científico orientado a mejorar la calidad y la justicia educativa de México y de la región latinoamericana. 